\title{
New Genus of the Actinomycetales: Actinosynnema gen. nov.
}

\author{
TORU HASEGAWA, $\dagger$ MARY P. LECHEVALIER, AND H. A. LECHEVALIER \\ Waksman Institute of Microbiology, Rutgers, The State University, Piscataway, New Jersey 08854
}

\begin{abstract}
An aerobic species of a new genus, Actinosynnema, is described under the name $A$. mirum sp. nov. The type strain of $A$. mirum is strain Hasegawa 101 (= IMRU 3971). The new genus is characterized by the formation of synnemata originating from the substrate mycelium. At the tip of the synnemata, chains of zoospores are produced.
\end{abstract}

Synnemata, also called coremia, are compacted groups of erect hyphae which are often fused and which bear conidia at their apex only or at both their apex and on their sides. Sterile columnar hyphal structures are sometimes also called synnemata or coremia.

The formation of synnemata and related structures in Actinomycetales is well documented. These have been reported in the genus Streptomyces by Krasil'nikov (Actinomyces) (11), Waksman (21), Grein (6), and Grein and Spalla (7), and "spore dome colonies" have been observed by Willoughby (22) in unidentified isolates of actinomycetes.

The present paper reports the description of an aerobic actinomycete that was isolated from a grass blade and which forms synnemata with zoospores. The new organism differs significantly from previously described species of actinomycetes and cannot be accommodated in any of the previously described genera of the actinomycetes. It is proposed to include it in a new genus, Actinosynnema, the type species of which is $A$. mirum. A culture of the type strain, Hasegawa 101, has been deposited in the culture collection of the Waksman Institute of Microbiology, Rutgers, The State University, Piscataway, N.J., under the number IMRU 3971.

\section{MATERIALS AND METHODS}

Bacterial strain. Strain 101 was isolated in September 1976 from a grass blade which was collected from the Raritan River, New Jersey, placed on yeast extract agar $(0.02 \%$ yeast extract [Difco], $1.5 \%$ agar), and incubated for 3 weeks at $28^{\circ} \mathrm{C}$. After that period of incubation, the surface of the agar was covered with various types of microbial growth, but, on the grass blade itself, small synnemata could be observed with a stereoscopic microscope. With a flamed loop, some of the synnemata were transferred to a sterile plate of the same medium from which the strain was isolated.

Media. The following media were used in this study: Bennett agar (9), tyrosine agar (18), yeast-malt agar

† Present address: Central Research Division, Takeda Chemical Industries Ltd., 17-85 Juso-honmachi 2 chome, Yodogawa-ku, Osaka 532, Japan.
(18), water agar (8), yeast-starch agar (1), Czapek agar (21), oatmeal agar (18), nutrient agar (Difco), thin Pablum agar (15), yeast-dextrose agar (21), glucoseasparagine agar (21), N-Z-amine glycerol agar (12), soil agar (12), inorganic salts-starch agar (18), thin potato-carrot agar (TPC) (8), and defined agar (12). The medium used for morphological observations was mainly TPC agar.

Microscopy. The study of whole mounts of sporophores by transmission electron microscope was done by the method of Lechevalier and Lechevalier (14) with a JEM-120 electron microscope operated at 80 $\mathrm{kV}$. For observation of zoospores, drops of the suspension containing zoospores were placed on Formvarcoated, 300-mesh copper grids. The excess liquid was absorbed on filter paper and was replaced by a drop of $2.0 \%$ phosphotungstic acid adjusted to $\mathrm{pH} 6.2$ with potassium hydroxide. The excess stain was removed with filter paper, and, after air drying, the preparations were promptly examined with the electron microscope.

For scanning electron microscopy, a TPC agar block with numerous synnemata was put on an aluminum stub and dried overnight in vacuo. The dried specimen was mounted with silver paint on the same stub and was then coated with gold. The coated specimen was examined in an Autoscan scanning electron microscope.

Other conditions of growth. Grass blades washed with sterile water were placed both in a moist chamber and on TPC agar. Spores of A. mirum were inoculated onto the surface of the grass blades and cultured for 1 week at $28^{\circ} \mathrm{C}$.

For growth tests both in a $4 \% \mathrm{CO}_{2}$ atmosphere and under anaerobic conditions, the GasPak systems (BBL) were used.

Physiological tests. The tests employed were those of Gordon and Mihm (5), and Gordon (3, 4); phosphatase production was estimated by the method of Barber and Kuper (2).

Cell wall and whole-cell analyses. Cell walls were analyzed by the method of Suput et al. (19), and whole cells were analyzed by the procedure of Lechevalier (13).

Staining procedures. The Gram stain for actinomycetes, in which aniline crystal violet is used as the primary stain, and the Kinyoun acid-fast stain modified for actinomycetes (16) were used.

Susceptibility to antibiotics and antimicrobial agents. The susceptibility of strain 101 to a few antibiotics and antimicrobial agents was examined by the 
following methods: (i) susceptibility disks (Difco) were placed onto Bennett agar and TPC agar seeded with a suspension of the test strain; after incubation for 1 week at $28^{\circ} \mathrm{C}$, the diameters of the inhibition zones were measured; (ii) antibiotics were incorporated into nutrient agar (Difco), and the test organisms were streaked onto the surface; after 1 week of incubation, the surface growth was compared with that on a plate without antibiotics (control)

Antibiotic properties. Antibiotic properties were studied by the cross -streak test on TPC and Bennett agars (20) and by the agar-dilution method. The strain was grown in four liquid media: Pablum (14), NYG-3 ( $8 \mathrm{~g}$ of nutrient broth [Difco], $10 \mathrm{ml}$ of glycerol, $1 \mathrm{~g}$ of yeast extract, $1 \mathrm{~g}$ of $\mathrm{NaNO}_{3}$, and $1,000 \mathrm{ml}$ of tap water), yeast-dextrose agar (20), and N-Z-amine glycerol agar (12). After 4 and 7 days the broths were filtered through paper and assayed by dilution in nutrient agar against four test organisms: Escherichia coli IMRU 54, Staphylococcus aureus IMRU 15, Pseudomonas sp. LL-NC2, and Candida albicans LL-204.

\section{RESULTS}

Description of Actinosynnema gen. nov. (From actis, actinos [Gr. noun], ray; syn [Gr. adv.], together; and nema, nematos [Gr. noun, neuter], thread.)

Fine hyphae (about $0.5 \mu \mathrm{m}$ in diameter) are produced which form a mycelium differentiated into: (i) a substrate mycelium that penetrates the agar and also grows into and forms synnemata or domelike bodies on the surface of agar; and (ii) an aerial mycelium that arises from the synnemata, domelike bodies, or flat colonies. The aerial hyphae bear chains of conidia capable of forming flagella in an aqueous environment. The cell wall is of type III, and the whole-cell sugar pattern is of type $\mathrm{C}$.

Type species: Actinosynnema mirum sp. nov. (from mirus, - $a$, -um [Lat. adj.], marvelous).

Substrate mycelium. The hyphae are about 0.5 to $1.0 \mu \mathrm{m}$ in diameter. Long, branching filaments penetrate the agar medium and form synnemata or domelike bodies that are at first whitish on most media and then become yellowish or yellowish orange.

Aerial mycelium. The hyphae are about 0.5 to $1.0 \mu \mathrm{m}$ in diameter. Long, branching hyphae, white to pale yellow, are produced, but not abundantly, on most media. Aerial mycelium is formed at the ends of synnemata on such agar media as TPC, tyrosine agar, and water agar. On yeast-malt agar, yeast-dextrose agar, Bennett agar, thin Pablum agar, and glucose-asparagine agar, aerial hyphae are formed both at the ends of synnemata or domelike bodies and on the surfaces of flat colonies. In young, growing cultures, the tips of the aerial hyphae often curl (Fig. 1).

Synnemata. Synnemata originate from the substrate mycelium and are formed on complex and synthetic media. TPC agar is the best medium found so far for the production of synnemata and the formation of the aerial mycelium at its tip (Fig. 2). Also, synnemata are formed on the surfaces of grass blades placed on agar media or in a moistened atmosphere. The size of a typical synnema formed on the TPC is illustrated in Fig. 3.

General microscopic appearance. The surface of a sporulating colony of $A$. mirum viewed perpendicularly with a light microscope appears covered with tufts of aerial hyphae. If the surface of the agar is tilted, the synnemal nature of the tufts is revealed (Fig. 4).

Spores. Spores are borne in chains. Septa are set down along the aerial hyphae and give rise to chains of spores. The septa are not apparent under the light microscope; however, in electron microscope preparations they appear as "swellings," which give the hyphae a bamboo-like appearance (Fig. 5 and 6). The spores are easily separated from each other. If the synnemata on TPC agar are placed on a slide and covered with brain heart infusion broth (Difco) and cover slips, motile spores can be observed after about $1 \mathrm{~h}$ at room temperature. The rod-shaped spores ( 0.4 to $1.0 \mu \mathrm{m}$ by 1.5 to $3.0 \mu \mathrm{m}$ ) are peritrichous and bear 5 to 15 flagella (Fig. 7). Moreover, motile elements are observed in other media such as static cultures in actinomyces broth (Difco) or TPC broth. During germination, one to three germ tubes are formed.

Cell chemistry. The cells have cell walls of type III containing meso-diaminopimelic acid in addition to alanine, glutamic acid, and glucosamine. Galactose and mannose are present in whole-cell hydrolysates. Madurose is absent. The organism can thus be considered to have a type $\mathrm{C}$ whole-cell sugar pattern.

Diffusible pigments. This strain produces a pale yellowish-brown soluble pigment on tyrosine agar and a pale greenish one on oatmeal agar. It does not produce any diffusible pigments on other media.

Appearance on various media. All cultures were incubated for 2 weeks at $28^{\circ} \mathrm{C}$. No growth took place on the defined medium.

Yeast-malt agar: Growth excellent, yellowish (color code Coo2a of Prauser [17]); synnemata moderate. Aerial mycelium poor, white.

Water agar: Growth moderate, whitish; synnemata numerous. Aerial mycelium moderate, white.

Yeast-starch agar: Growth moderate, yellowish (Coo3a); synnemata numerous. Aerial mycelium poor, white.

Tyrosine agar: Growth moderate, yellowish 


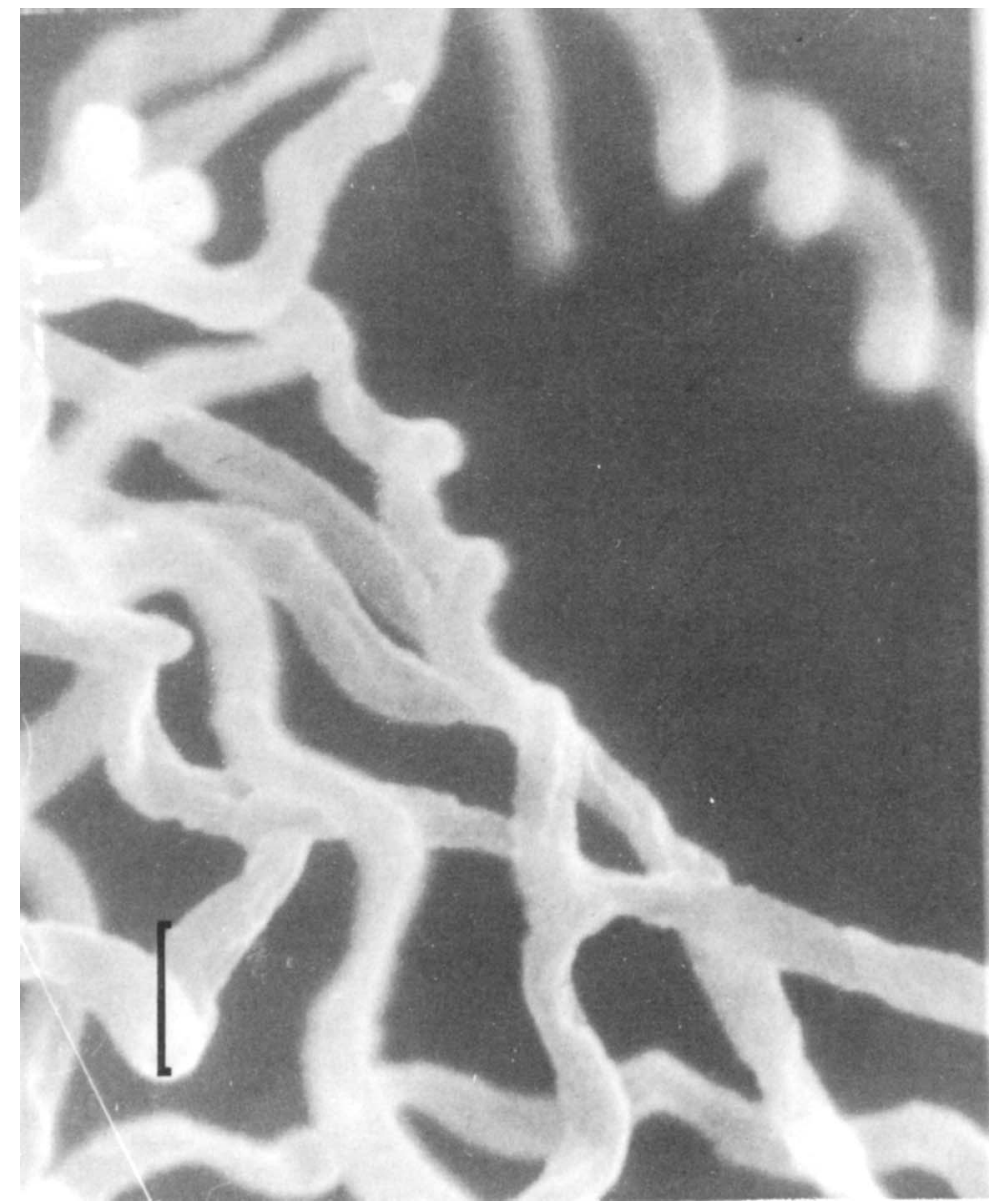

FIG. 1. Scanning electron micrograph of aerial hyphae at the tip of a synnema. Bar represents $1 \mu \mathrm{m}$. TPC agar, 5-day-old culture.

(Coo2a); synnemata numerous. Aerial mycelium moderate, white. Soluble pigment pale yellowish brown.

Czapek agar: Growth moderate, yellowish (Coo2a); dome-like bodies few. Aerial mycelium poor, white.

Bennett agar: Growth excellent, yellowish (Coo2a-Coo2r); synnemata and domelike bodies numerous. Aerial mycelium moderate, white.

Oatmeal agar: Growth excellent, yellowish (Coo4a-Coo2a); synnemata few. Aerial mycelium poor, white. Soluble pigment pale greenish yellow (Co4r).

Nutrient agar: Growth moderate, yellowish (Coo2a); synnemata few. No aerial mycelium.

Thin Pablum agar: Growth moderate, yellowish (Coo2a); synnemata numerous. Aerial mycelium moderate, white.

Yeast-dextrose agar: Growth moderate, yellowish (Coo2a); synnemata numerous. Aerial mycelium poor, white.
Glucose-asparagine agar: Growth moderate, yellowish (Coo2a); synnemata and domelike bodies moderate. Aerial mycelium moderate, white.

N-Z-amine glycerol agar: Growth moderate, orange-yellow; glistening, domelike bodies are moderate; synnemata few. Aerial mycelium poor, white.

Soil agar: Growth poor, whitish, thin; synnemata few. No aerial mycelium.

Inorganic salts-starch agar: Growth poor, whitish; synnemata few. Aerial mycelium poor, white.

TPC agar: Growth moderate, yellowish (Coo2a); synnemata numerous. Aerial mycelium moderate, white.

Staining characteristics. The hyphae and spores are mainly gram positive and are not acid fast.

Physiological and biochemical characteristics. No growth took place on any medium 


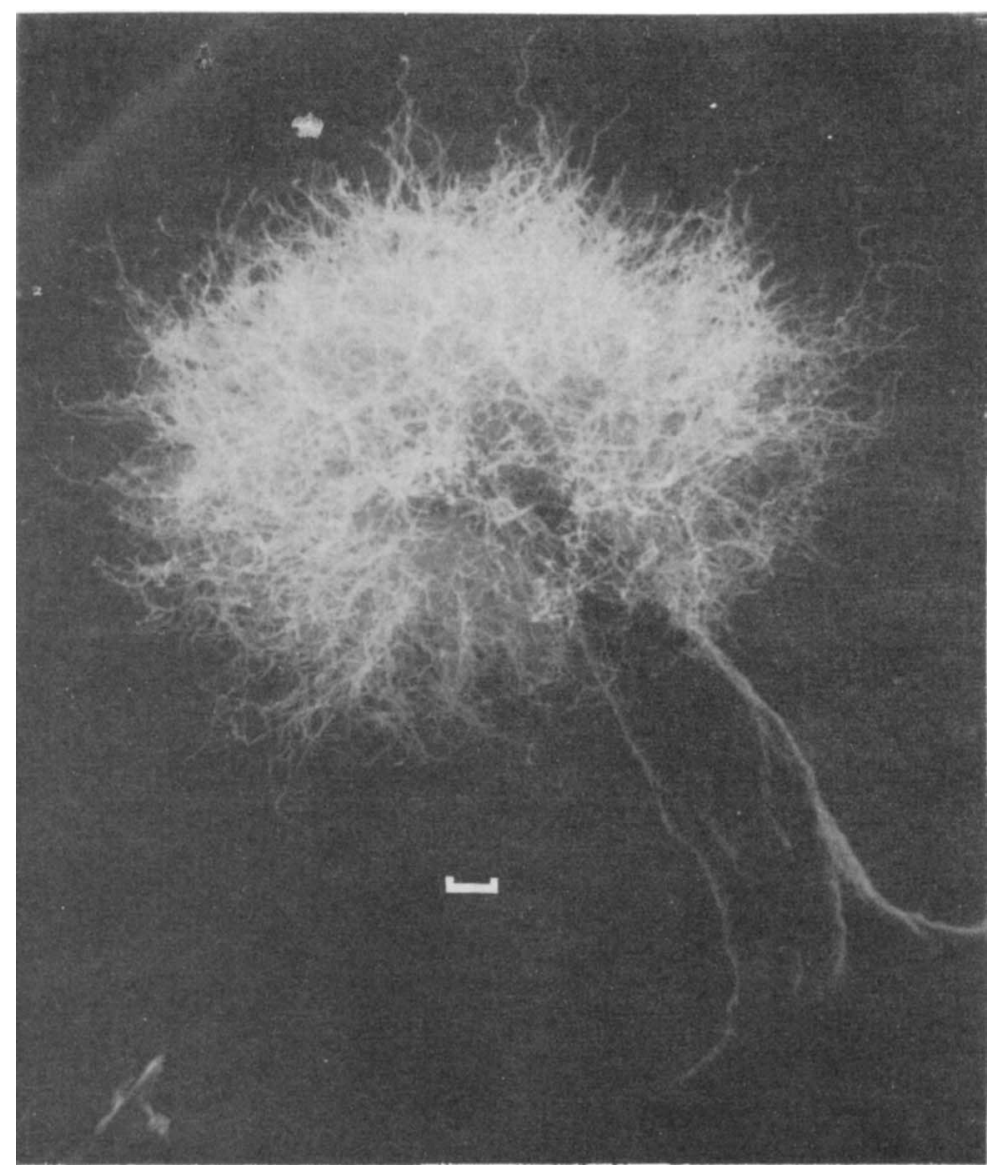

FIG. 2. Scanning electron micrograph of a synnema. Bar represents $10 \mu \mathrm{m}$.

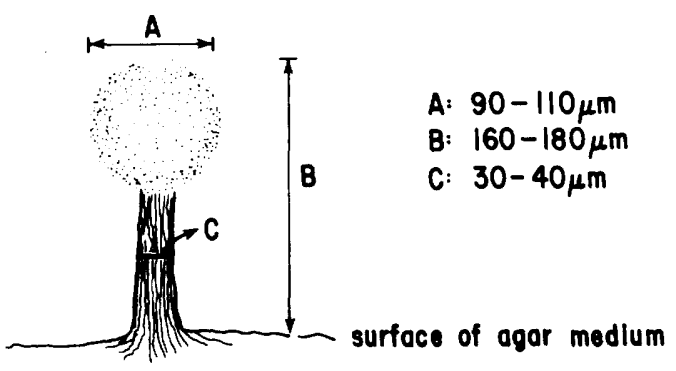

FIG. 3. Diagram representing the dimension of $a$ synnema of A. mirum grown on TPC agar.

under anaerobic conditions, but growth was moderate under a $\mathrm{CO}_{2}$ atmosphere. The temperature range for growth was 10 to $35^{\circ} \mathrm{C}$. Starch, casein, tyrosine, and gelatin were hydrolyzed, but xanthine, hypoxanthine, adenine, and urea were not. Nitrate reductase and phosphatase were produced. Milk was peptonized. Growth was produced in lysozyme broth. No growth occurred at $37^{\circ} \mathrm{C}$ or on $5 \% \mathrm{NaCl}$. Tartrate, py- ruvate, lactate, and malate were utilized. Benzoate, acetate, citrate, and succinate were not utilized. Acid was produced from fructose, lactose, maltose, D-mannitol, L-arabinose, D-melibiose, D-mannose, L-rhamnose, xylose, dextrin, galactose, glucose, trehalose, raffinose, soluble starch, sucrose, cellobiose, glycogen, and adonitol. No acid was produced from $i$-inositol, sorbitol, D-ribose, salicin, inulin, glycerol, dulcitol, erythritol, $\alpha$-methyl-D-glucoside, or $\alpha$-methylD-mannoside.

Antibiotic properties. Zones of inhibition by cross-streak test were produced with grampositive bacteria (Bacillus megaterium LL-CW 4A, $10 \mathrm{~mm}$; Sarcina lutea IMRU 14, $23 \mathrm{~mm}$ ), a mycobacterium ( $M$. smegmatis ATCC 607, 8 $\mathrm{mm}$ ), filamentous fungi (Aspergillus niger LL13, $25 \mathrm{~mm}$ [incomplete inhibition]; Penicillium notatum LL-40, $25 \mathrm{~mm}$ [incomplete inhibition]) and yeasts (Candida tropicalis LL-206, $10 \mathrm{~mm}$; Saccharomyces cerevisiae LL-216, $10 \mathrm{~mm}$ ). Shake-flask cultures showed no activity except for a slight activity against $C$. albicans in the 


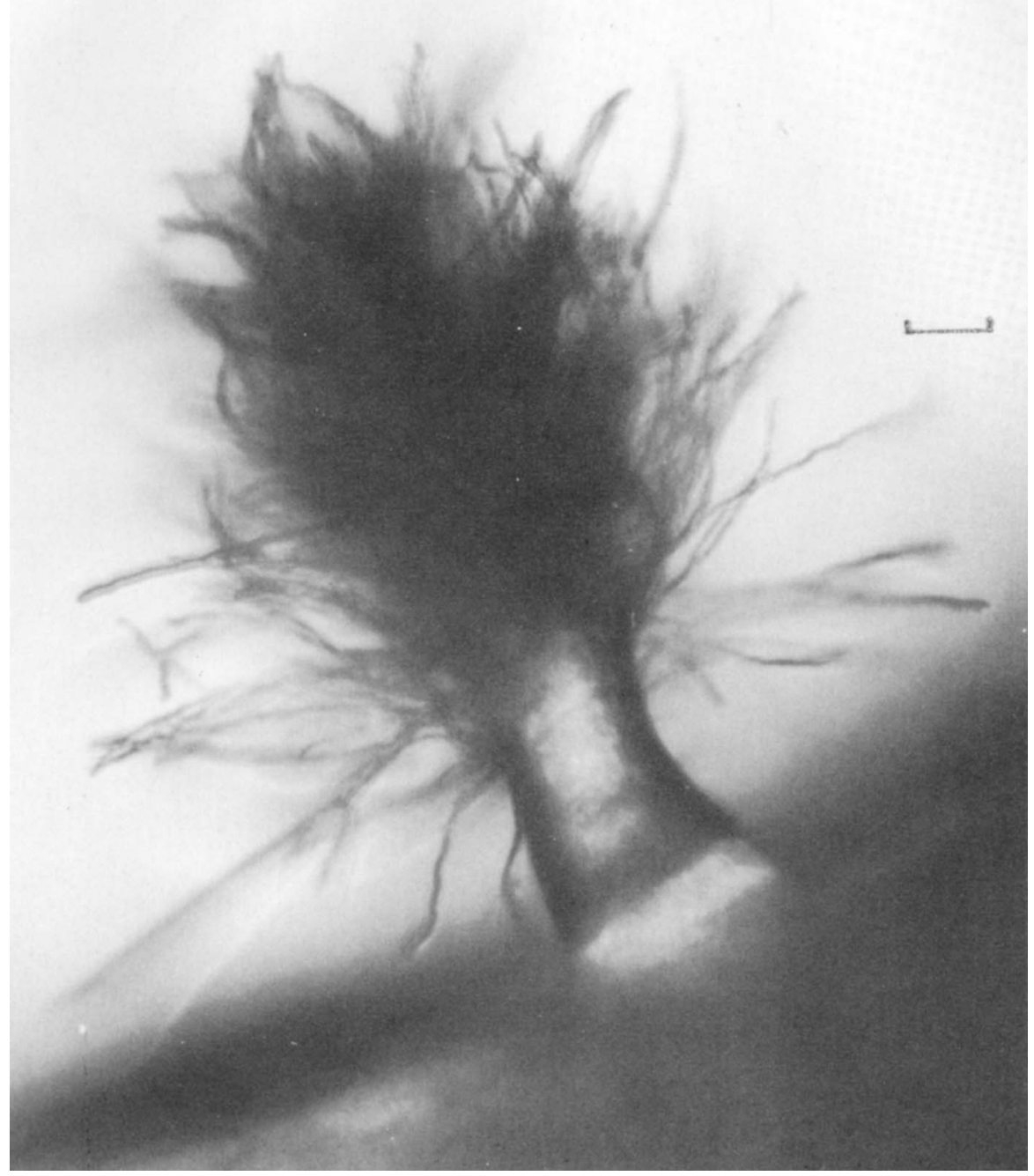

FIG. 4. Micrograph of a synnema growing on a plate of TPC agar. The surface of the agar was slanted to visualize the stem of the synnema. Bright-field microscopy. Bar represents $10 \mu \mathrm{m}$.

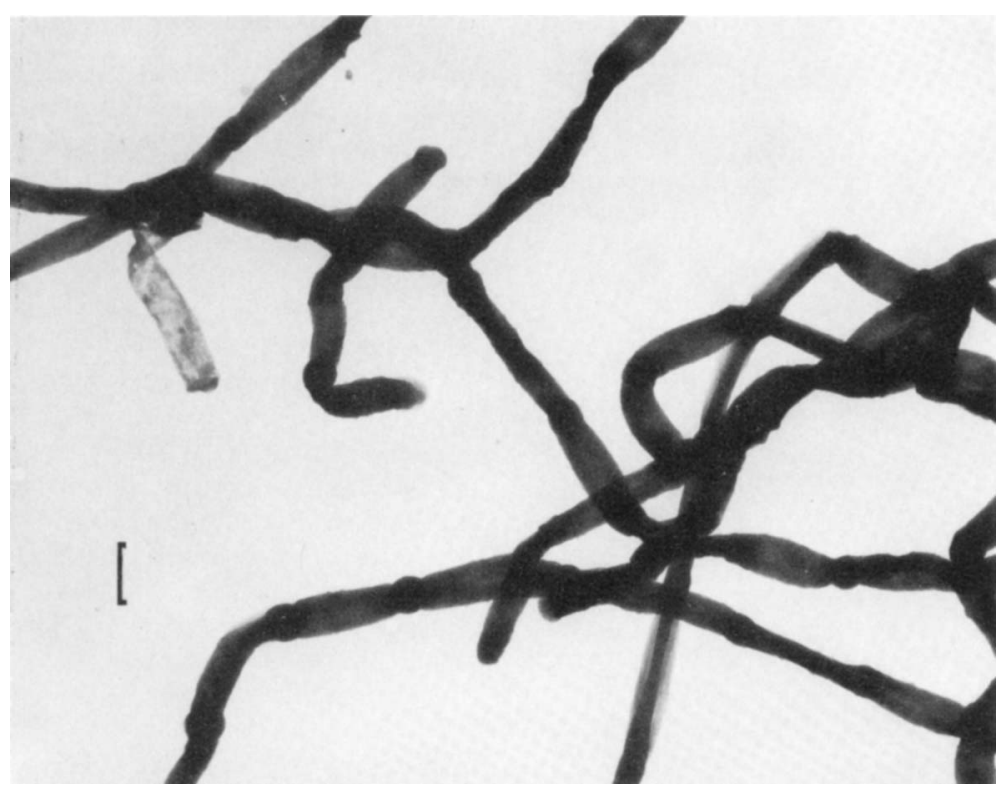

FIG. 5. Electron micrograph of spore chains from the tip of a synnema. TPC agar, 7-day-old culture. Bar represents $1 \mu \mathrm{m}$. 
case of Pablum broth (10 dilution units per $\mathrm{ml}$ ).

Susceptibility to antibiotics and antimicrobial agents. On nutrient agar, this organism grew in the presence of $50 \mu \mathrm{g}$ of candicidin per $\mathrm{ml}$ and $100 \mu \mathrm{g}$ of nystatin per ml. Dihydrostreptomycin at $10 \mu \mathrm{g} / \mathrm{ml}$ completely inhibited growth as did $1 \mu \mathrm{g}$ of tetracycline per ml. By the diffusion method, growth was inhibited strongly by disks impregnated with tetracycline and its derivatives $(30 \mu \mathrm{g})$, chloramphenicol $(30 \mu \mathrm{g})$, novobiocin $(30 \mu \mathrm{g})$, and bacitracin $(10 \mu \mathrm{g})$.

Type strain. The type strain is Hasegawa 101 (= IMRU 3971). Because the species de-

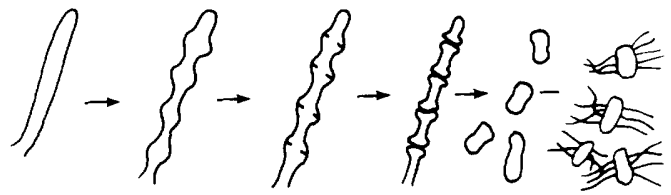

Fig. 6. Probable mode of spore formation in Actinosynnema mirum. scription given above is based on a single strain, the type strain, it also serves as the description of the type strain.

\section{DISCUSSION}

In a recent review, Kalakoutskii and Agre (10) noted that synnemata are known to be produced by many actinomycetes but that no signs of special transformations of hyphae in these structures have been reported so far. We propose that organisms that form synnemata bearing, at their tips, chains of spores capable of forming flagella (when in an aqueous environment) be placed in the genus Actinosynnema.

It is difficult to place the genus Actinosynnema in any of the currently recognized families of the order Actinomycetales. Aerial chains of motile spores are formed in the genus Sporichthya, but the two genera can be differentiated on the basis of formation of substrate mycelium and synnemata in the genus Actinosynnema and

FIG. 7. Electron micrograph of a zoospore of $A$. mirum. Bar represents $0.1 \mu \mathrm{m}$. 
their absence in the genus Sporichthya. In addition, members of these two genera are very different in their cell wall compositions (type I for Sporichthya, type III for Actinosynnema).

A number of actinomycetes with a type III cell wall are known to produce motile elements. These include the sporangia-forming, madurosecontaining members of the genera Planomonospora, Planobispora, and Spirillospora. Actinomycetes of this spore wall type with motile elements which do not form sporangia include the madurose-containing members of the genus Dermatophilus and their relatives in the genus Geodermatophilus which lack madurose. However, there is no morphological similarity between the Dermatophilaceae, which do not form aerial hyphae but which produce masses of motile cocci by the division of hyphae, and $A$. mirum with its aerial chains of motile, rodshaped spores.

Pending the isolation of more strains of actinomycetes forming synnemata with chains of spores at the tip of these structures, we feel that the genus Actinosynnema should be considered one of the genera of the order Actinomycetales which are of uncertain familial placement.

\section{ACKNOWLEDGMENTS}

This work was supported by National Science Foundation grant DEB 73-01692. The senior author was the holder of the Waksman-Merck Postdoctoral Fellowship when this work was done.

We thank Robert Albert Holzer for observation with the scanning electron microscope and Fusao Kondo for advice on transmission electron microscopy.

\section{REPRINT REQUESTS}

Address reprint requests to: H. A. Lechevalier, Waksman Institute of Microbiology, Rutgers University, P. O. Box 759, Piscataway, NJ 08854

\section{LITERATURE CITED}

1. Arai, T. 1975. Culture media for actinomycetes. The Society for Actinomycetes, Japan.

2. Barber, M., and S. W. A. Kuper. 1951. Identification of Staphylococcus pyogenes by the phosphatase reaction. J. Pathol. Bacteriol. 63:65-68.

3. Gordon, R. E. 1966. Some criteria for the recognition of Nocardia madurae. J. Gen. Microbiol. 45:355-364.

4. Gordon, R. E. 1967. The taxonomy of soil bacteria, p.
293-321. In T. Gray, R. G. Parkinson, and B. Parkinson (ed.), The ecology of soil bacteria. Liverpool University Press, Liverpool, England.

5. Gordon, R. E., and J. M. Mihm. 1962. Identification of Nocardia caviae (Erikson) nov. comb. Ann. N.Y. Acad. Sci. 98:628-636.

6. Grein, A. 1968. Description of a new species of Streptomyces, Streptomyces racemosus, characterized by an unusual morphological structure. G. Microbiol. 16:1-8.

7. Grein, A., and C. Spalla. 1962. Studio sui coremi formati in culture di Streptomyces peucetius. G. Microbiol. 10:175-187.

8. Higgins, M. L., M. P. Lechevalier, and H. A. Lechevalier. 1967. Flagellated actinomycetes. J. Bacteriol. 93:1446-1451.

9. Jones, K. L. 1949. Fresh isolates of actinomyctes in which the presence of sporogenous aerial mycelia is a fluctuating characteristic. J. Bacteriol. 57:141-145.

10. Kalakoutskii, L. V., and N. S. Agre. 1976. Comparative aspects of development and differentiation in actinomycetes. Bacteriol. Rev. 40:469-524.

11. Krasil'nikov, N. A. 1938. Ray fungi and related organisms. Actinomycetales. Publishing House of the U.S.S.R. Academy of Science, Moscow.

12. Lechevalier, H. A., M. Solotorovsky, and C. I. McDurmont. 1961. A new genus of the actinomycetales: Micropolyspora gen. nov. J. Gen. Microbiol. 26:11-18.

13. Lechevalier, M. P. 1968. Identification of aerobic actinomycetes of clinical importance. J. Lab. Clin. Med. 71:934-944.

14. Lechevalier, M. P., and H. A. Lechevalier. 1957. A new genus of actinomycetales: Waksmania gen. nov. J. Gen. Microbiol. 17:104-111.

15. Lechevalier, M. P., H. A. Lechevalier, and P. E. Holbert. 1967. Sporichthya, un nouveau genre de Streptomycetaceae. Ann. Inst. Pasteur (Paris) 114:277-286.

16. Park, G. 1970. Reagents, stains, and test procedures, p. 675-692. In J. E. Blair, E. H. Lennette, and J. P. Truant (ed.), Manual of clinical microbiology. American Society for Microbiology, Bethesda, Md.

17. Prauser, H. 1964. Aptness and application of colour codes for exact description of colours of streptomycetes. Z. Allg. Mikrobiol. 4:95-98.

18. Shirling, E. B., and D. Gottlieb. 1966. Methods for characterization of Streptomyces species. Int. J. Syst. Bacteriol. 16:313-340.

19. Suput, J., M. P. Lechevalier, and H. A. Lechevalier. 1967. Chemical composition of variants of aerobic actinomycetes. Appl. Microbiol. 15:1356-1361.

20. Waksman, S. A. 1950 . The actinomycetes-their nature, occurrence, activities, and importance. Chronica Botanica Co., Waltham, Mass.

21. Waksman, S. A. 1961. The actinomycetes, vol. II. The Williams and Wilkins Co., Baltimore.

22. Willoughby, L. G. 1969. A study on aquatic actinomycetes. The allochthonous leaf component. Nova Hedwigia 18:45-113. 